\title{
Enramado y riego como alternativas de rehabilitación de regiones semiáridas afectadas por el tendido de ductos
}

\author{
Esteban Kowaljow ${ }^{1, \otimes}$ \& César M. RostagnO ${ }^{2}$ \\ 1. Instituto Multidisciplinario de Biología Vegetal (IMBIV). CONICET-Universidad Nacional de Córdoba. 2. Centro Nacional \\ Patagónico (CENPAT). CONICET.
}

\begin{abstract}
RESUMEN. La instalación subterránea de cañerías requiere de la remoción de la vegetación y la compactación del suelo debido al tránsito de maquinaria pesada. Evaluamos el efecto de la aplicación de ramas de "quilembai" (Chuquiraga avellanedae Lorentz) sobre algunas propiedades físicas y químicas del suelo y sobre el establecimiento de la vegetación, simulando la construcción de "islas de fertilidad", características del monte Patagónico. Seleccionamos dos sitios que sufrieron diferentes disturbios en un área afectada por la instalación de un gasoducto: i) desmonte, decapitación del suelo superficial y circulación de maquinaria pesada (Zona 1), y ii) desmonte, movimiento y deposición del suelo y la vegetación removida (Zona 2). En cada sitio se aplicaron tratamientos de agregado de ramas en dos dosis distintas que a su vez recibieron, o no, riego. También implementamos un tratamiento control y otro que solo recibió riego. Diez meses después de la aplicación se determinó humedad del suelo, densidad aparente, resistencia a la penetración, velocidad de infiltración, C orgánico y P-Olsen y se cuantificó la densidad de plantas, antes de la aplicación y a los 10 meses. Los tratamientos aumentaron la humedad del suelo respecto al control, aunque sólo en algunos casos lo hicieron en forma significativa; no se observaron tendencias claras en el resto de las variables edáficas. La densidad de gramíneas, principalmente Nassella tenuis (Phil.) Barkworth, aumentó en los tratamientos con ramas de la Zona 1, y se observaron efectos positivos tanto de la dosis como del riego. No se detectó un efecto significativo de los tratamientos en la densidad de gramíneas en la Zona 2. En el corto plazo, la aplicación de ramas y riego en sitios muy disturbados no modificó significativamente las características físicas y químicas del suelo, no obstante se observó un aumento notable en el establecimiento de gramíneas, acelerando la recuperación de la cobertura vegetal.
\end{abstract}

[Palabras clave: compactación, establecimiento de la vegetación, gasoducto, paquetes de ramas, Patagonia, propiedades del suelo]

\begin{abstract}
Shrub packs and irrigation to rehabilitate semiarid regions disturbed by the laying of pipelines: The installation of underground pipelines generally requires the removal of the vegetation cover and has the potential, along with the heavy traffic associated with the installation, to affect the soil quality. In this study we assessed the effect of application of "quilembai" (Chuquiraga avellanedae Lorentz) branches on selected soil properties and vegetation recovery in two sites disturbed by the installation of a pipeline: i) an area where the vegetation and topsoil were removed and affected by heavy machinery traffic (Zone 1), and ii) an area where the vegetation and topsoil were removed and, after the pipeline was installed, they were redistributed over the disturbed area (Zone 2). Branches were applied at two application rates, without or with irrigation. There was also an irrigation treatment and a control. Ten months after the treatments were applied, soil water content, bulk density penetration resistance, infiltration rate, soil organic $C$ and Olsen- $P$ were determined. The density of plants in each plot was determined at the beginning and 10-months post treatment application. The treatment application increased soil water content, although with a varying effect among zones and we did not detect treatment effects on other soil variables. Plant density, mainly that of Nassella tenuis (Phil.) Barkworth, increased in all the treatments with branches of the zone 1, with positive effects of application rates as well as irrigation, while zone 2 did not reveal effects of treatments. Although in 10-months post-application the quilembai branches and irrigation had no significant effects on the soil physical and chemical properties, they increased plant establishment, accelerating the pace of vegetation recovery.
\end{abstract}

[Keywords: compaction, vegetation establishment, gas pipeline, brushpacks, Patagonia, soil properties]

\section{INTRODUCCIÓN}

La presión sobre los ecosistemas áridos y semiáridos está aumentando debido a la mayor demanda de servicios tales como alimentos, fibras, forraje, combustible, agua y materiales de construcción. Este aumento

$\triangle$ Instituto Multidisciplinario de Biología Vegetal (IMBIV). CONICET-Universidad Nacional de Córdoba. Córdoba, Argentina.

ekowaljow@gmail.com se atribuye a una combinación de factores humanos (e.g., la presión demográfica y los cambios en el uso de la tierra) y climáticos (e.g., las sequías). Las actividades como el pastoreo, la minería, la extracción de petróleo, la construcción de carreteras y la instalación de cañerías, modifican la estructura de estos

Recibido: 24 de julio de 2012; Fin de arbitraje: 24 de agosto; Versión revisada: 4 de septiembre, Aceptado: 20 de noviembre 
frágiles ecosistemas a través de disminuir su cobertura vegetal y de acelerar los procesos de degradación de suelos. El desmonte, seguido por el tráfico o el pisoteo, puede resultar en un suelo suelto en superficie e inestabilidad de dunas, aumento en la erosión del suelo, disminución de la productividad y otros efectos similares (Ci \& Yang 2010).

Ante este panorama, y debido a que en muchos casos la recuperación natural de la integridad de los ecosistemas de estas regiones se ve limitada por las elevadas temperaturas, el viento, la escasa humedad y la baja fertilidad del suelo, se han planteado diferentes estrategias de restauración y rehabilitación (Bainbridge 2007).

A menudo, la vegetación en los sistemas áridos y semiáridos se distribuye como un conjunto de parches de plantas, insertados en una matriz de suelo desnudo o con muy baja cobertura. Estos parches, también llamados "islas de fertilidad", pueden capturar y retener agua, sedimentos, propágulos y nutrientes provenientes de las áreas desnudas (Rostagno et al. 1991; Valentin et al. 1999). Por este motivo, algunas técnicas de rehabilitación se basan en la recuperación de estos parches o facilitan la formación de parches artificiales. En regiones semiáridas del este de Australia, Ludwig \& Tongway (1996) observaron que, luego de tres años, la aplicación de ramas de Acacia aneura sobre la superficie del suelo simulando parches de vegetación produjo micrositios fértiles que aumentaron el establecimiento, el crecimiento y la supervivencia de diferentes plantas perennes. En pastizales áridos y semiáridos de Sudáfrica, esta técnica de rehabilitación, también llamada "enramado", "paquetes de ramas" o "brushpacks", aumentó la humedad del suelo y el reclutamiento natural de especies anuales (Simons \& Allsopp 2007; Hanke et al. 2011). Estos resultados indicarían que la aplicación de ramas produce un microambiente benéfico para el desarrollo de algunas especies vegetales, disminuyendo en forma indirecta la erosión del suelo.

En un trabajo previo (Kowaljow \& Rostagno 2008) describimos el impacto del tendido de un gasoducto y de la reposición parcial del material removido sobre algunas propiedades físicas y químicas del suelo y sobre la cobertura vegetal en el monte Patagónico. Las principales conclusiones fueron que: i) la mayor limitación de los suelos disturbados fue su baja capacidad para incorporar agua, derivada principalmente de su compactación elevada y su baja porosidad (lo que produciría una lenta recuperación de la cobertura vegetal), y ii) la reposición del material removido por la actividad del desmonte no significó una medida de recuperación eficaz.

El objetivo del presente trabajo fue evaluar el efecto de la aplicación de ramas de Chuquiraga avellanedae y de riegos complementarios sobre algunas propiedades físicas y químicas del suelo y sobre la recuperación de la vegetación en un área del monte Patagónico que fue afectada por el tendido de un gasoducto. Se evaluaron dos situaciones: un sitio donde se decapitó el suelo y se compactó por la circulación de maquinaria pesada (Zona $1)$, y un sitio donde, luego del desmonte, se repuso el suelo y el material vegetal removido (Zona 2). Planteamos las siguientes hipótesis y predicciones: i) Las pilas de ramas, al favorecer la captura de propágulos y mantillo y la incorporación de materia orgánica, mejoran las propiedades físicas de los suelos, favoreciendo un mayor ingreso y retención de humedad. Bajo estas condiciones se prevé un mayor desarrollo de la vegetación; ii) las pilas de ramas, al disminuir el impacto de las gotas de agua sobre el suelo, evitan la formación de costras y aumentan la retención de humedad. Como consecuencia de ello, los tratamientos con riego tendrán un efecto positivo en la recuperación de la vegetación cuando se apliquen conjuntamente con las pilas de ramas; iii) el efecto de las pilas de ramas y el riego sobre el suelo y la vegetación, será mayor a mayor grado de disturbio (Zona 1).

\section{Materiales y Métodos}

\section{Área de estudio}

El área de estudio se encuentra en el noreste de la provincia de Chubut, Argentina. La precipitación varía entre 150 y $220 \mathrm{~mm} /$ año y la temperatura media anual es $13.5^{\circ} \mathrm{C}$ (Barros \& Rivero 1982). Los suelos dominantes son de textura areno franca y han sido clasificado como Calciorthides y Paleorthides (Rostagno \& del Valle 1988). El área de estudio está ubicada en el extremo sur de la Provincia Fitogeográfica del Monte (Cabrera 1953). La vegetación es una estepa arbustiva en la que dominan arbustos tales como jarilla (Larrea divaricata Cav.), brusquilla (Prosopidastrum globosum (Gillies ex Hook. \& Arn.) Burkart), quilembai (Chuquiraga avellanedae Lorentz) y molle (Schinus johnstonii F.A. Barkley), con un tapiz herbáceo compuesto mayormente por flechilla (Nassella tenuis (Phil.) 
Barkworth), coirón poa (Poa ligularis Nees ex Steud. var. ligularis) y coirón amargo (Pappostipa speciosa (Trin. \& Rupr. Romasch.) (Beeskow et al.1987).

En el mes de marzo de 1997, la Empresa Transportadora de Gas del Sur S.A. (TGS) construyó un gasoducto de $60 \mathrm{~km}$ de largo, desde el gasoducto principal hasta el parque industrial de la ciudad de Puerto Madryn. Previo a la instalación del gasoducto, se produjo el desmonte y el decapitamiento de los 10-15 cm superficiales de suelo, en una franja de $16 \mathrm{~m}$ de ancho. En el sector central de la franja desmontada se abrió una zanja de $1 \mathrm{~m}$ de ancho por $1 \mathrm{~m}$ de profundidad en la que se instaló la cañería. Luego, tanto el suelo que se extrajo de esta zanja como el que fue removido de los horizontes superficiales junto a los restos de la vegetación removida, fueron depositados en una franja de $\sim 6 \mathrm{~m}$ de ancho (Zona 2); el sector restante se utilizó para el tránsito de camiones y máquinas pesadas (Zona 1) (Figura 1).

\section{Diseño del experimento}

En septiembre de 1999 (2 años y medio después del tendido del gasoducto) en las Zonas 1 y 2 se delimitaron dos áreas de $125 \mathrm{~m}^{2}$ (5x25m). En cada área fueron instaladas 30 parcelas de $1 \mathrm{~m}^{2}$. Los 6 tratamientos fueron asignados al azar a las parcelas $(n=5)$. Se aplicaron los siguientes tratamientos: i) aplicación de una lámina de agua de $20 \mathrm{~mm}$ cada 30 días (riego=R), ii) $1.35 \mathrm{~kg}$ de ramas de C. avellanedae por $\mathrm{m}^{2}$ (M1), iii) $2.7 \mathrm{~kg}$ de ramas de C. avellanedae por $\left.\mathrm{m}^{2}(\mathrm{M} 2), \mathrm{iv}\right) 1.35 \mathrm{~kg}$ de ramas más riego $(\mathrm{M} 1+\mathrm{R})$, v) $2.7 \mathrm{~kg}$ de ramas más riego $(\mathrm{M} 2+\mathrm{R})$, y vi) un tratamiento control sin riego ni aplicación de ramas (C). Las ramas, de $1 \mathrm{~cm}$ de diámetro y $20-30 \mathrm{~cm}$ de largo, fueron cortadas a mano a partir de individuos de $C$. avellanedae verdes en pie que se encontraban en los alrededores del área experimental. A cada arbusto se le extrajo un máximo de $30 \%$ de sus ramas con el fin de evitar daños severos. Las ramas se distribuyeron en toda la superficie de la parcela y fueron cubiertas con una red (malla de $8 \mathrm{~cm}$ ) para evitar que las removiera el viento. En el sector donde se instaló el experimento, el gasoducto se extiende de oeste a este, en el sentido de los vientos predominantes, lo que habría favorecido una mayor movilidad de los propágulos. Las precipitaciones se registraron en forma mensual y fueron comparadas con los promedios históricos de la región.

\section{Variables analizadas y metodología de análisis}

A los 10 meses de aplicados los tratamientos, en cada parcela de $1 \mathrm{~m}^{2}$ se determinó la densidad aparente de los 0-5 cm superficiales de suelo con la técnica del sacabocados (Blake 1965), la resistencia a la penetración en los $5 \mathrm{~cm}$ superficiales de suelo utilizando un penetrómetro de cono (Davidson 1965), y la velocidad de infiltración del suelo por el método del cilindro (Bertrand 1965). En muestras de suelos de los 0-5 cm superficiales, tamizadas por $2 \mathrm{~mm}$, se determinó humedad por gravimetría. En estas se determinó el contenido de $C$ orgánico por el procedimiento de Walkley y Black (Nelson \& Sommers 1982) y el contenido de P extraíble con bicarbonato de sodio por colorimetría (Olsen \& Sommers 1982). En un sector no disturbado cercano al gasoducto (ecosistema de referencia), se seleccionaron cinco "islas de fertilidad" o sectores debajo de los arbustos, de aquí en adelante montículos $(\mathrm{M})$ y las áreas adyacentes o sectores entre arbustos, de aquí en adelante entremontículos (EM). En ambos sectores se tomaron muestras de suelo $(0-5 \mathrm{~cm})$, a las que se le realizaron las mismas determinaciones físicas y químicas del suelo $(n=5)$. Los datos obtenidos del área de referencia no fueron utilizados en el análisis estadístico.

En cada parcela de $1 \mathrm{~m}^{2}$ se determinó la densidad de gramíneas antes y 10 meses después de la aplicación de los tratamientos (Mueller-Dombois \& Ellenberg 1974). Las gramíneas se clasificaron en 5 categorías: 1 (menos de 5 hojas), 2 (entre 6 y 12 hojas), 3 ( $>12$ hojas y $<2 \mathrm{~cm}$ de diámetro basal),

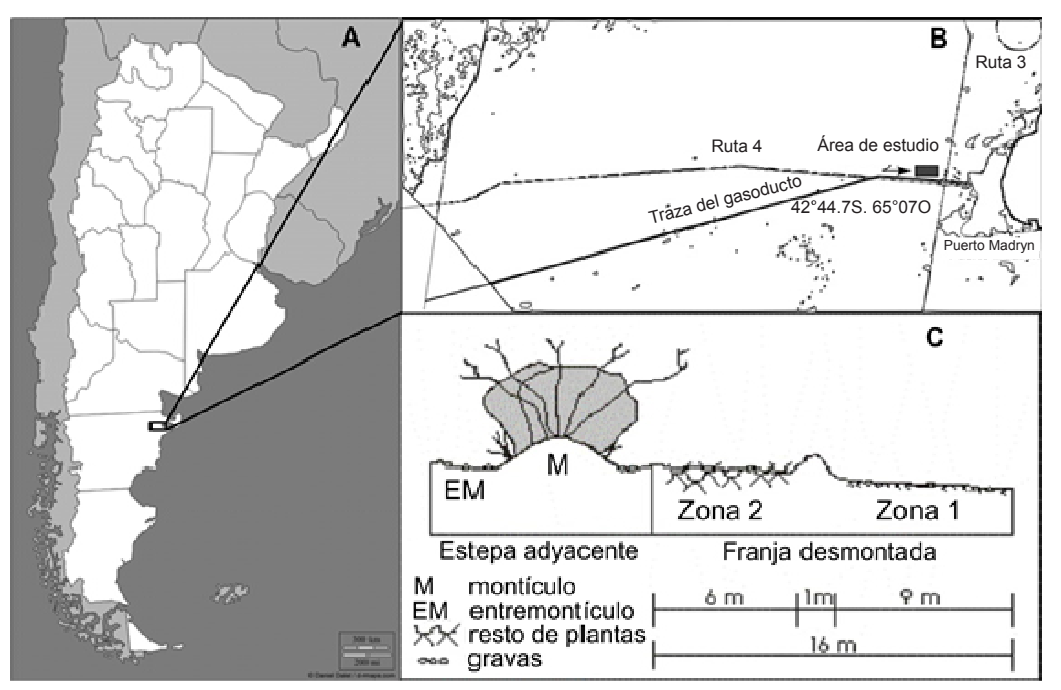

Figura 1. Ubicación geográfica del área de estudio (A y B) y detalle de un corte transversal de la traza del gasoducto (C). Se identifican las zonas donde se removió la vegetación (Zonas 1 y 2), el camellón del gasoducto y la estepa adyacente donde se diferencia el suelo del montículo (M) y el suelo entre montículo (EM).

Figure 1. Geographic location of the study area (A and B) and detail of a cross section of pipeline trace (C). Details as in legend in Spanish. 
4 (entre 2 y $5 \mathrm{~cm}$ de diámetro basal), y 5 (>5 cm de diámetro basal).

Para determinar la significancia estadística de los tratamientos se realizó, en cada zona por separado, análisis de la varianza (ANOVA). Cuando los tratamientos tuvieron diferencias significativas $(P<0.05)$ se realizó una separación de medias empleando la Diferencia Significativa Menor (LSD; Steel \& Torrie 1980). Se utilizó el programa Statistica (StatSoft 1984-2003).

\section{Resultados}

El registro de precipitaciones mostró que durante los 10 meses que duró el experimento llovió $254 \mathrm{~mm}$, valor superior al promedio anual de precipitaciones de la región (229 mm) (período 1977-2007). La distribución de las precipitaciones durante el experimento varió en relación a los datos históricos, con un fuerte máximo en primavera en lugar de los picos más frecuentes de fin del verano e inicio del otoño (Figura 2).

No se observó un efecto importante de los tratamientos en las variables edáficas evaluadas. Se observó un aumento en el contenido de humedad del suelo en los tratamientos con ramas y con ramas y riego para ambas zonas (Tabla 1). Resultaron significativas las diferencias respecto al control en $\mathrm{M} 1+\mathrm{R}$ para la Zona 1, y M1, $\mathrm{M} 1+\mathrm{R}$ y M2 para la Zona 2. Si bien el resto de los tratamientos no mostraron diferencias significativas con el control, se observó un ligero aumento en la humedad del suelo en los tratamientos con ramas y con ramas y riego (Tabla 1). También se observaron diferencias

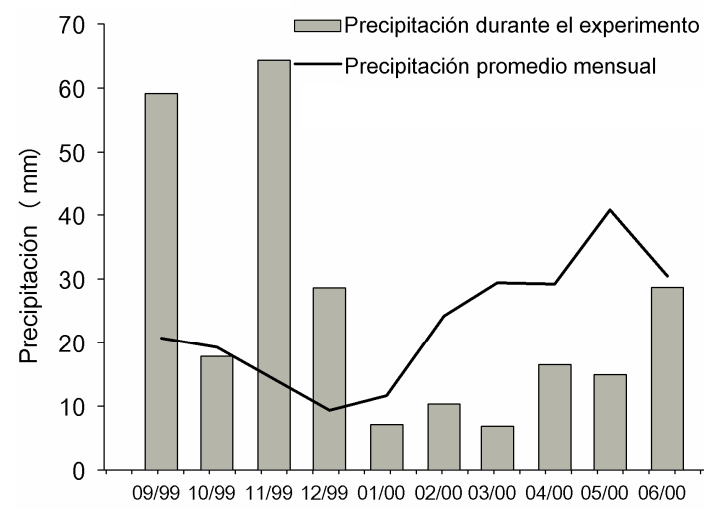

Figura 2. Precipitaciones ocurridas durante el experimento (barras) y promedio de precipitaciones histórico de la región (línea continua, intervalo 1977-2007).

Figure 2. Rainfalls during the experiment (bars) and historical average rainfall for the region (solid line, range 1977-2007).

significativas en el contenido de $C$ del suelo en la Zona 2 (Tabla 1), pero en este caso no hubo diferencias de los tratamientos respecto al control y no se observó una tendencia clara. En la Zona 1, las parcelas que solo fueron regadas mostraron una menor velocidad de infiltración y contenido de nutrientes; de todas maneras no se observaron diferencias significativas con el control (Tabla 1). En el área de referencia se encontró una marcada diferencia entre suelo del montículo y el entremontículo, teniendo el suelo del montículo mejores propiedades físico-químicas. Los suelos de la Zona 1 se asemejaron más al suelo del entremontículo, si bien presentaron mayor resistencia a la penetración y menor velocidad de infiltración. Por otro lado, los valores de densidad aparente y en menor medida de $\mathrm{C}$ orgánico

Tabla 1. Características físicas y químicas del suelo en los diferentes tratamientos y el área de referencia. Diferentes letras indican diferencias significativas entre los tratamientos para cada zona $(P<0.05)$. C: control; R: riego; M1: 1.35 $\mathrm{kg}$ de ramas por $\mathrm{m}^{2} ; \mathrm{M} 1+\mathrm{R}: 1.35 \mathrm{~kg}$ de ramas con riego; M2: $2.7 \mathrm{~kg}$ de por $\mathrm{m}^{2} ; \mathrm{M} 2+\mathrm{R}: 2.7 \mathrm{~kg}$ de ramas con riego; $\mathrm{M}$ : montículo; EM: entremontículo; DA: densidad aparente; RP: resistencia a la penetración; Infiltración: velocidad de infiltración; s/d: sin datos.

Table 1. Soil physical and chemical properties in treatments and reference area. Different letters indicate significant differences among treatments for each zone $(P<0.05)$. Details as in legend in Spanish.

\begin{tabular}{|c|c|c|c|c|c|c|c|c|c|}
\hline & \multicolumn{6}{|c|}{ Tratamientos } & \multicolumn{2}{|c|}{$\begin{array}{l}\text { Área de } \\
\text { referencia }\end{array}$} \\
\hline & & C & $\mathrm{R}$ & M1 & $\mathrm{M} 1+\mathrm{R}$ & M2 & $\mathrm{M} 2+\mathrm{R}$ & $\mathrm{M}$ & EM \\
\hline \multirow{2}{*}{ Humedad (\%) } & Zona 1 & $5.7 \mathrm{a}$ & $5.1 \mathrm{a}$ & $7.1 \mathrm{ab}$ & $7.4 \mathrm{~b}$ & $7.0 \mathrm{ab}$ & $6.0 \mathrm{ab}$ & \multirow{2}{*}{$\mathrm{s} / \mathrm{d}$} & \multirow{2}{*}{$\mathrm{s} / \mathrm{d}$} \\
\hline & Zona 2 & $4.1 \mathrm{a}$ & $5.3 \mathrm{ab}$ & $9.8 \mathrm{~b}$ & $10.5 \mathrm{~b}$ & $7.2 \mathrm{~b}$ & $7.2 \mathrm{ab}$ & & \\
\hline \multirow{2}{*}{$\mathrm{DA}\left(\mathrm{g} / \mathrm{cm}^{3}\right)$} & Zona 1 & $1.4 \mathrm{a}$ & $1.4 \mathrm{a}$ & $1.4 \mathrm{a}$ & $1.4 \mathrm{a}$ & $1.3 \mathrm{a}$ & $1.3 \mathrm{a}$ & \multirow{2}{*}{1.1} & \multirow{2}{*}{1.5} \\
\hline & Zona 2 & $1.2 \mathrm{a}$ & $1.1 \mathrm{a}$ & $1.1 \mathrm{a}$ & $1.1 \mathrm{a}$ & $1.0 \mathrm{a}$ & $1.1 \mathrm{a}$ & & \\
\hline \multirow{2}{*}{$\mathrm{RP}(\mathrm{MPa})$} & Zona 1 & $1.2 \mathrm{a}$ & $1.2 \mathrm{a}$ & $1.2 \mathrm{a}$ & $1.3 \mathrm{a}$ & $1.2 \mathrm{a}$ & $1.2 \mathrm{a}$ & \multirow[b]{2}{*}{0.3} & \multirow{2}{*}{0.9} \\
\hline & Zona 2 & $0.9 \mathrm{a}$ & $1.1 \mathrm{a}$ & $1.0 \mathrm{a}$ & $1.0 \mathrm{a}$ & $1.0 \mathrm{a}$ & $1.0 \mathrm{a}$ & & \\
\hline \multirow{2}{*}{ Infiltración (mm/h) } & Zona 1 & $39.2 \mathrm{a}$ & $26.5 \mathrm{a}$ & $28.2 \mathrm{a}$ & $32.4 \mathrm{a}$ & $44.6 \mathrm{a}$ & $36.6 \mathrm{a}$ & \multirow{2}{*}{403} & \multirow{2}{*}{61} \\
\hline & Zona 2 & $189.8 \mathrm{a}$ & $167.2 \mathrm{a}$ & $155.0 \mathrm{a}$ & $152.8 \mathrm{a}$ & $201.1 \mathrm{a}$ & $203.4 \mathrm{a}$ & & \\
\hline \multirow{2}{*}{ C orgánico $(\mathrm{g} / \mathrm{kg})$} & Zona 1 & $4.5 \mathrm{a}$ & $4.2 \mathrm{a}$ & $5.5 \mathrm{a}$ & $5.1 \mathrm{a}$ & $5.2 \mathrm{a}$ & $5.3 \mathrm{a}$ & \multirow{2}{*}{11} & \multirow{2}{*}{4} \\
\hline & Zona 2 & $8.3 \mathrm{ab}$ & $7.3 \mathrm{ab}$ & $4.9 \mathrm{ab}$ & $4.7 \mathrm{~b}$ & $8.0 \mathrm{ab}$ & $10.6 \mathrm{a}$ & & \\
\hline \multirow{2}{*}{ P extraíble (mg/kg) } & Zona 1 & $6.7 \mathrm{a}$ & $3.9 \mathrm{a}$ & $5.5 \mathrm{a}$ & $5.1 \mathrm{a}$ & $5.1 \mathrm{a}$ & $5.0 \mathrm{a}$ & \multirow{2}{*}{8.3} & \multirow{2}{*}{4.7} \\
\hline & Zona 2 & $6.2 \mathrm{a}$ & $4.9 \mathrm{a}$ & $6.8 \mathrm{a}$ & $5.3 \mathrm{a}$ & $8.0 \mathrm{a}$ & $10.1 \mathrm{a}$ & & \\
\hline
\end{tabular}


y P extractable de los suelos de la Zona 2, se asemejaron a los del montículo (Tabla 1).
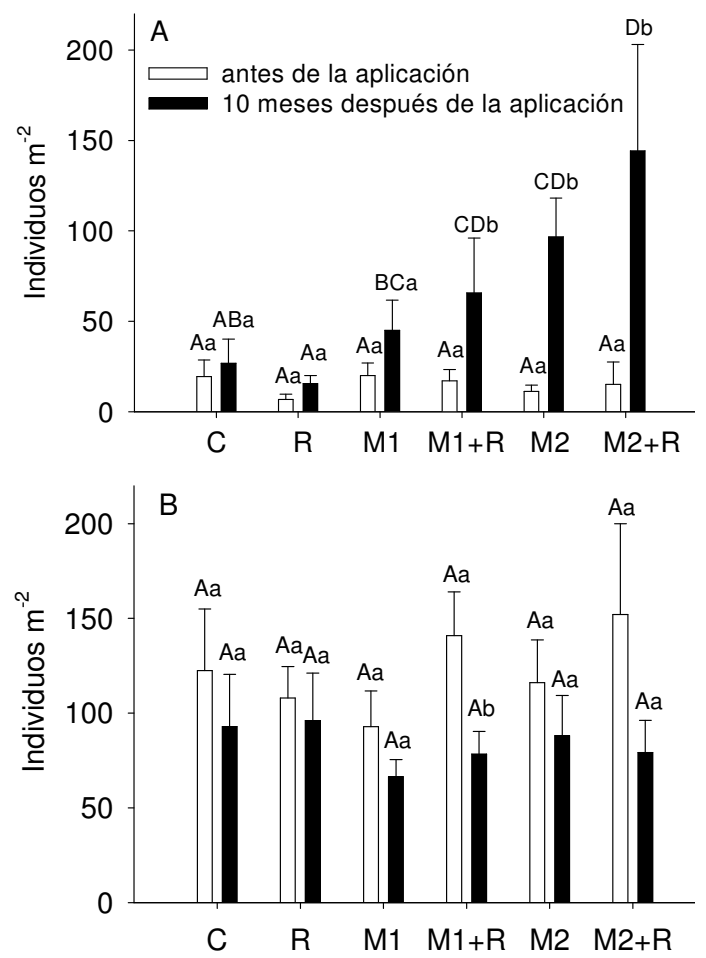

Figura 3. Densidad de gramíneas en la Zona 1 (A) y en la Zona 2 (B), antes y 10 meses después de la aplicación de las ramas y el riego. Diferentes letras mayúsculas indican diferencias significativas entre tratamientos $(P<0.05)$. Diferentes letras minúsculas indican diferencias significativas entre parcelas para el mismo tratamiento, antes y 10 meses después de la aplicación $(P<0.05)$. Los códigos de los tratamientos se detallan en la Tabla 1.

Figure 3. Grass density in zone 1 (A) and zone 2 (B) before and 10 months after application of the branches and irrigation. Details as in legend in Spanish.
Las gramíneas (anuales y perennes) fueron el grupo dominante en ambas zonas. En el tiempo que duró el experimento, las hierbas latifoliadas anuales no fueron comunes ni se relacionaron con la aplicación de los diferentes tratamientos. La hierba latifoliada perenne más frecuente fue Noticastrum sericeum (Less.) Less. ex Phil. La densidad de gramíneas en la Zona 1 aumentó de forma notable en todos los tratamientos con ramas (sólo significativo para M1+R, M2 y M2+R), y se observaron efectos positivos tanto de la dosis de ramas como del riego suplementario (Figura $3 \mathrm{~A}$ ). Este aumento se debió principalmente al establecimiento de nuevas plántulas de $N$. tenuis, herbácea dominante en la estepa adyacente a la zona disturbada (Figura 4). Si bien las diferencias no fueron significativas, en la Zona 2 se observó una disminución en la densidad de gramíneas al final del experimento. Esta tendencia se observa para todos los tratamientos, inclusive el tratamiento control (Figura 3B).

\section{DisCUSIÓN}

La falta de respuesta de la mayoría de las variables edáficas evaluadas a los tratamientos con ramas y ramas con riego se podría atribuir a diferentes factores. Uno de ellos es que el tiempo transcurrido desde la aplicación de los tratamientos (10 meses) pudo no haber sido suficiente para que resultaran afectadas las características de los suelos debajo de los parches de ramas. De acuerdo al modelo de desarrollo post-disturbio postulado por Eldridge \& Green (1994), el mejoramiento en las propiedades físicas de los suelos comienza a producirse luego del establecimiento de algunas plantas anuales y perennes. Por lo
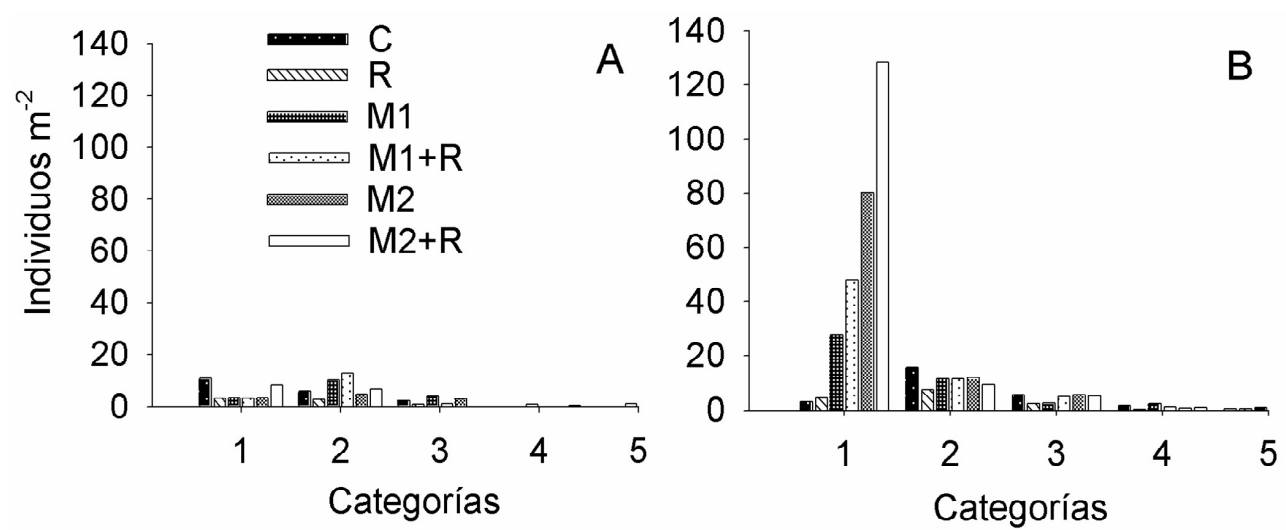

Figura 4. Densidad de gramíneas en la Zona 1 agrupados en cinco categorías de tamaño, antes (A) y 10 meses después (B) de la aplicación de las ramas y el riego. Los códigos de los tratamientos se detallan en la Tabla 1.

Figure 4. Grass density in zone 1 grouped in five size categories, before (A) and 10 months after (B) application of the branches and irrigation. Codes of the treatments are shown in Table 1. 
general, las plantas perennes favorecen el desarrollo estructural del suelo y aumentan la porosidad e infiltrabilidad de los suelos degradados. En nuestro estudio, si bien se registró la instalación de una abundante cantidad de plantas, la gran mayoría correspondían a plantas recientemente establecidas. Tongway \& Ludwig (1996) observaron un efecto positivo de la aplicación de ramas sobre el contenido de nutrientes en suelos de textura fina, muy compactos y de muy baja fertilidad, luego de tres años de su aplicación. Por otro lado, estos mismos autores observaron que el aumento de la mayoría de los nutrientes del suelo, debajo de parches de ramas, era evidente sólo en el primer centímetro de suelo y no en los intervalos de 1 a 3,3 a 5 y 5 a $10 \mathrm{~cm}$, mientras que nuestros resultados se refieren a los primeros $5 \mathrm{~cm}$ de suelo.

A pesar del escaso tiempo transcurrido desde la aplicación y la profundidad de suelo utilizada $(5 \mathrm{~cm})$, los tratamientos con ramas y ramas con riego en ambas zonas tendieron a aumentar el contenido de humedad del suelo. Esta tendencia estuvo muy relacionada a la presencia de ramas ya que el tratamiento con riego y sin ramas de la Zona 1 mostró el menor contenido de humedad. Estos resultados coinciden en forma parcial con los obtenidos por Hanke y colaboradores (2011) en estepas arbustivas degradadas de Sudáfrica desarrolladas sobre suelos poco profundos, de textura areno-franco. Estos autores observaron que luego de 11 meses, los parches con ramas de arbustos aumentaron el contenido y la capacidad de retención de agua del suelo, produciendo una disminución de la desecación en los primeros $5 \mathrm{~cm}$ de suelo.

Por lo general, la presencia de mantillo modifica el microambiente de los suelos de los pastizales (Willms et al. 1986) al incrementar la infiltración del agua, reducir la incidencia de la radiación solar y aislar la superficie del suelo. De esta manera se mantienen temperaturas más bajas en la estación cálida, lo que reduce la evaporación (Dormaar \& Willms 1992). Sería importante de todas maneras, profundizar en este aspecto en futuros trabajos, ya que en los sistemas áridos y semiáridos la disponibilidad de agua regula en gran medida la dinámica de la vegetación y de los ciclos biogeoquímicos (Noy-Meir 1973; Austin et al. 2004). La falta de una tendencia clara en el efecto de los tratamientos en el contenido de C del suelo en la Zona 2 probablemente se deba a un efecto de la heterogeneidad de los suelos de este sitio como resultado de la redeposición del suelo removido, sin tener en cuenta si los horizontes eran superficiales o sub-superficiales.

En la Zona 1, los tratamientos con ramas aumentaron de manera considerable el establecimiento de gramíneas, probablemente debido a la mayor captura de propágulos y a las condiciones microambientales. Las diferencias observadas entre tratamientos sugieren que la cantidad de ramas es una variable a tener en cuenta en esta metodología de rehabilitación, ya que en los tratamientos con mayor dosis de ramas $\left(2.7 \mathrm{~kg}\right.$ de ramas $\left./ \mathrm{m}^{2}\right)$ se registró el mayor número de plántulas. Por otro lado también se observó una tendencia a que el riego aplicado en forma conjunta con las ramas aumenta el establecimiento de plántulas. En un trabajo reciente Simons \& Allsopp (2007) observaron que luego de 2 años, la aplicación de ramas aumentó la cobertura vegetal. Aste aumento se debió a un mayor establecimiento de especies de ciclo anual. Los autores sostienen que el establecimiento de anuales serviría como una protección del suelo desnudo y que con el tiempo podría aumentar el contenido de materia orgánica del suelo. Sin embargo, concluyen que si bien el reclutamiento natural estuvo afectado de forma positiva por la presencia de ramas, no se observó el mismo efecto respecto a su supervivencia. Lamentablemente, en el presente trabajo no se pudo evaluar la supervivencia de plántulas debido a que el área experimental fue afectada en forma involuntaria durante las tareas de mantenimiento del gasoducto.

Otras técnicas de restauración como el excavado de surcos u hoyos, la aplicación de fertilizantes inorgánicos, o la siembra, tuvieron solo un éxito parcial en diferentes regiones, aun bajo buenas condiciones ambientales (Green 1989; Pressland \& Graham 1989; Simons \& Allsopp 2007; Hanke et al. 2011). Por otro lado, la aplicación de enmiendas orgánicas y la plantación de especies vegetales surgen como otras alternativas viables para la rehabilitación de ambientes degradados (Kowaljow \& Mazzarino 2007; Yüksek \& Yüksek 2011). El enramado presenta la ventaja de utilizar un material que, en general, se encuentra cerca del área a recuperar. También mejora las condiciones microambientales al favorecer el desarrollo de la vegetación; probablemente, en el largo plazo aumente la estructura, el contenido de nutrientes y la materia orgánica del suelo. Por otro lado, si la superficie a 
recuperar es de dimensiones considerables, se debería evaluar su impacto en la vegetación de la cual se obtienen las ramas.

Distintos autores han discutido sobre la importancia de recuperar la composición, la estructura y la funcionalidad del ecosistema cuando se plantean estrategias de restauración en regiones áridas y semiáridas (Maestre et al. 2006; Cortina et al. 2006). Según estos autores, en sitios con síntomas claros de disminución funcional, las acciones de restauración deberían focalizarse en aumentar la estabilidad del suelo, la infiltración y el ciclado de nutrientes. En este caso sería recomendable la aplicación de métodos de bajo costo como la creación de parches con ramas de arbustos (Ludwig \& Togway 1996; Tongway \& Ludwig 1996). En sitios con mejor situación funcional, las acciones de restauración deberían hacer foco en, por ejemplo, la introducción de nuevas especies con el objeto de mejorar las funciones ecosistémicas, incrementar la resiliencia ante otros disturbios y fomentar el establecimiento de plantas y la recuperación de las poblaciones de animales (Maestre \& Cortina 2004). Si bien la metodología de rehabilitación utilizada en el presente trabajo no mejoró de forma significativa los atributos edáficos, sí tuvo un efecto positivo sobre el reclutamiento y la germinación de propágulos en el ambiente más degradado (Zona 1). Por otro lado, en el sitio menos disturbado (Zona 2) no se observó este efecto sobre la vegetación. Para acelerar la recuperación de este sitio se deberían realizar otro tipo de intervenciones, como la plantación de arbustos.

\section{CONCLUSIONES}

Luego de 10 meses de su aplicación, las pilas de ramas tendieron a aumentar el contenido de agua del suelo y aumentaron el establecimiento de plantas en el sitio más disturbado (Zona 1). El riego no es una estrategia de rehabilitación efectiva, ya que solo aumentó el establecimiento de especies vegetales cuando se aplicó conjuntamente con las pilas de ramas. En la región semiárida de Patagonia, las pilas de ramas surgen como una opción viable en la rehabilitación de sitios disturbados que hayan perdido cobertura vegetal, nutrientes y capacidad de infiltración de agua del suelo.

\section{BIBLIOGRAFÍA}

Austin, AT; L Yahditan; JM Stark; J Belnap; A Porporato; ET AL. 2004. Water pulses and biogeochemical cycles in arid and semiarid ecosystems. Oecologia, 141:221-235.
BAInBridge, DA. 2007. A guide for desert and dryland restoration: a new hope for arid lands. Island Press, Washington, DC, USA. Pp. 347.

BArros, V \& MM Rivero. 1982. Mapas de probabilidad de precipitación de la provincia del Chubut. Contribución número 54, Centro Nacional Patagónico, Puerto Madryn, Chubut, Argentina. Pp. 25.

BeesKow, AM; HF del Valle \& CM Rostagno. 1987. Los sistemas fisiográficos de la región árida y semiárida de la provincia de Chubut. Secretaría de Ciencia y Técnica, Delegación Regional Patagonia. S. C. Bariloche, Río Negro, Argentina. Pp. 168.

Bertrand, AR. 1965. Rate of Water Intake in the fiel. Pp. 197-209 en: Black, CA (ed.). Methods of Soil Analysis. Part 1. American Society of Agronomy, Madison, Winsconsin, USA.

BlaKe, B. 1965. Bulk density. Pp. 374-390 en: Black, CA (ed.). Methods of Soil Analysis. Part 1. American Society of Agronomy, Madison, Winsconsin, USA.

CABRERA, AL. 1953. Esquema fitogeográfico de la República Argentina. Revista del Museo de La Plata, Botánica, 8: 87-168.

CI, L \& X YANG. 2010. Desertification and its control en China. Higher Education Press, Beijing. Springer, Heidelberg, Dordrecht, London, New York. Pp. 507.

Cortina, J; FT Maestre; R Vallejo; MJ Baeza, A VALDECANTOS; ET AL. 2006. Ecosystem structure, function, and restoration success: Are they related? J. Nat. Conserv., 14:152-160.

Davidson, DT. 1965. Penetrometer Measurements. Pp. 472-484 en: Black, CA (ed.). Methods of Soil Analysis. Part 1. American Society of Agronomy, Madison, Winsconsin, USA.

DORMAAR, JF \& WD WiLlms. 1992. Water-extractable organic matter from plant litter and soil of rough fescue grassland. J. Range Management, 45:152-158.

GREEN, DR. 1989. Rangeland restoration projects in western New South Wales. Aust. Rang. J., 11:110-116.

ELDRIDGE, DJ \& RSB GREEN. 1994. Microbiotic soil crusts - a review of their roles in soil and ecological processes in the rangelands of Australia. Aust. J. Soil Res., 32: 389-415.

HANKE, W; A GRÖNGRÖFT; N JÜRGENS \& U SCHMIEDEL. 2011. Rehabilitation of arid rangelands: intensifying water pulses from low-intensity winter rainfall. J. Arid Environ., 75:185-193.

Kowaljow, E \& CM Rostagno. 2008. Efectos de la instalación de un gasoducto sobre algunas propiedades del suelo superficial y la cobertura vegetal en el NE de Chubut. Ciencia del Suelo, 26:51-62.

Kowaljow, E \& MJ Mazzarino. 2007. Soil restoration in semiarid Patagonia: Chemical and biological response to different compost quality. Soil Biol. Biochem., 39: 1580-1588.

LUDWIG, JA \& DJ TONGWAY. 1996. Rehabilitation of Semiarid Landscapes in Australia. II. Restoring Vegetation Patches. Restor. Ecol., 4:398-406.

Maestre, FT \& J CortinA. 2004. Insights into ecosystem composition and function in a sequence of degraded semiarid steppes. Restor. Ecol., 12:494-502.

Maestre, FT; J Cortina \& R VAllejo. 2006. Are Ecosystem Composition, Structure, and Functional Status Related to Restoration Success? A Test from Semiarid Mediterranean Steppes. Restor. Ecol., 14:258-266. 
Mueller-Dombois, D \& H Ellenberg (eds.). 1974. Aims and methods of vegetation ecology. Wiley, New York, USA.

NeLSON, DW \& LE SOMmers. 1982. Total carbon, organic carbon, and organic matter. Pp. 539-579 en: Page, AL (ed.). Methods of Soil Analysis, Part 2. $2^{\mathrm{da}}$ ed. American Society of Agronomy, Madison, Winsconsin, USA.

NoY-MeIR, I. 1973. Desert ecosystems: environment and producers. Annu. Rev. Ecol. Syst., 4:25-52.

OLSEN, SR \& LE SOMmers. 1982. Phosphorus. Pp. 403-430 en: Page, AL (ed.). Methods of Soil Analysis, Part 2. 2da ed. American Society of Agronomy, Madison, Winsconsin, USA.

Pressland, AJ \& TWG Graham. 1989. Approaches to the restoration of rangelands-the Queensland experience. Aust. Rang. J., 11:101-109.

Rostagno, CM \& HF del Valle. 1988. Mounds associated with shrubs in aridic soils of north-eastern Patagonia: Characteristics and probable genesis. Catena, 15:347359.

Rostagno, CM; HF del Valle \& L Videla. 1991. The influence of shrubs on some chemical and physical properties of an aridic soil in north-eastern Patagonia,
Argentina. J. Arid Environ., 20:179-188.

SimONS, L \& N AlLsopr. 2007. Rehabilitation of Rangelands in Paulshoek, Namaqualand: Understanding vegetation change using biophysical manipulations. J. Arid Environ., 70:755-766.

StatSoft. 1984-2003. Statistica Release 6.1. StatSoft, Inc., Tulsa, USA.

Steel, R \& J Torrie. 1980. Principles and procedures of statistics. A biometrical approach. 2nd ed. McGraw-Hill, New York, USA.

TongWAY, DJ \& JA LuDWIG. 1996. Rehabilitation of Semiarid Landscapes in Australia. I. Restoring Productive Soil Patches. Restor. Ecol., 4:388-397.

Valentin, C; JM d'Herbes \& J Poesen. 1999. Soil and water components of banded vegetation patterns. Catena, 37 : $1-24$

Willms, WD; S SmoliaK \& AW Bafley. 1986. Herbage production following litter removal on Alberta native grasslands. J. Range Manage., 39:536-540.

YÜKSEK, T \& F YÜKSEK. 2011. The effects of restoration on soil properties in degraded land in the semi-arid region of Turkey. Catena, 84:47-53. 